\title{
Associations between anthropometric characteristics and insulin markers in mothers and their neonates and with neonate's birth weight: An observational cohort study
}

\author{
Ali Naseh ${ }^{1}$, Sormeh Nourbakhsh ${ }^{1}$, Maryam Tohidi², Paymaneh Sarkhail², Bita Najafian ${ }^{3}$, \\ Fereidoun Azizi² \\ ${ }^{1}$ Department of Pediatrics, Taleghani Hospital, and ${ }^{2}$ Research Institute for Endocrine Sciences, Shahid-Beheshti University \\ of Medical Sciences, Tehran, Iran; ${ }^{3}$ Department of Pediatrics, Baqiatallah Medical University, Tehran, Iran. \\ E-mail: tohidi@endocrine.ac.ir \\ Received: 8th March 2017, Accepted: 11th April 2017
}

SUMMARY: Naseh A, Nourbakhsh S, Tohidi M, Sarkhail P, Najafian B, Azizi F. Associations between anthropometric characteristics and insulin markers in mothers and their neonates and with neonate's birth weight: An observational cohort study. Turk J Pediatr 2017; 59: 625-635.

This study aimed to identify possible associations between anthropometric characteristics and insulin markers of mothers and 1) their neonate's birth weight, and 2) those markers of neonates. A prospective observational cohort of 100 healthy mothers who came to a hospital in Tehran in 2014 from pregnancy to delivery as well as their term neonates comprised the study population. Only newborns with weight within normal range were included. Anthropometric indices and serum glucose and insulin levels were measured in both mothers and neonates. Correlations between maternal body and serum indices and neonate's serum indices and birth weight were assessed. Maternal weight before pregnancy $(r=0.3, p=0.001)$, at time of delivery $(r=$ $0.3, \mathrm{p}=0.001)$, and maternal body mass index $(\mathrm{BMI})$ before pregnancy $(\mathrm{r}=0.2$, $\mathrm{p}=0.04$ ) positively associated with neonate's birth weight. For the neonates with normal birth weight, there was no correlation between maternal serum glucose and insulin levels and neonate's serum glucose and insulin levels or birth weight. Neonate's serum glucose correlated positively with insulin levels $(\mathrm{r}=0.3, \mathrm{p}=0.006)$ and HOMA-IR $(\mathrm{r}=0.6, \mathrm{p}<0.0001)$; and negatively with HOMA-S $(r=-0.6, p<0.0001)$ and QUICKI $(r=-0.5, p<0.0001)$. Neonate's insulin correlated positively with HOMA-IR $(\mathrm{r}=0.9, \mathrm{p}<0.0001)$, and negatively with HOMA-S $(\mathrm{r}=-0.9, \mathrm{p}<0.0001)$, QUICKI $(\mathrm{r}=-0.9, \mathrm{p}<0.0001)$, gestational age $(r=-0.2, p=0.03)$ and with glucose-insulin $(\mathrm{GI})$ ratio $(\mathrm{r}=-0.9, \mathrm{p}<0.0001)$. Neonate's GI ratio correlated positively with gestational age $(r=0.2, p=0.01)$. Maternal serum glucose and insulin showed positive correlation $(\mathrm{r}=0.4, \mathrm{p}$ $<0.0001)$. The lowest maternal insulin quartile had dominantly male and the highest quartile had dominantly female neonates $(p=0.006)$. In conclusion, maternal anthropometric measures correlate with neonates' birth weight. Advancing health promotion to normalize these maternal parameters may reduce the incidence of abnormal birth weights among newborns.

Key words: birth weight, maternal anthropometric characteristics, homeostatic model assessment, insulin resistance, insulin sensitivity.

Identifying the factors which determine infant's birth weight (BW) may enable the health service providers to develop preventive measures to reduce the incidence of low $\mathrm{BW}$ or macrosomia in newborns.

Newborns with birth weight below 10th percentile or 2,500 grams of weight are defined as small for gestational age (SGA). ${ }^{1}$ Also, based on the current available data, 2.3 to 10 percent of all live births consist of SGA newborns. ${ }^{2,3,4}$ Large for gestational age (LGA) includes newborns weighting at/over 4,000 grams and appropriate for gestational age (AGA) includes newborns who weigh 2,500 to less than 4,000 
grams. 5,6 Some studies showed that most neonates with SGA condition will appropriately catch up with their growth during their first two years of lives. However, $15 \%$ will stay with short stature state for life. ${ }^{7}$ Newborns with the SGA condition are in higher risk for neonatal death incidences and their future health may be more affected by coronary arterial disease, neuro-psychological problems, and metabolic diseases. ${ }^{8}$ Newborns with SGA condition are in higher risk for diabetes in their adulthood. ${ }^{9}$

Babies with SGA also have higher risk of developing obesity, insulin resistance, impaired glucose tolerance, and increased dyslipidemia. ${ }^{10,11}$ Additionally, the SGA condition affects the insulin-like growth factorgrowth hormone (IGF-GH) pathway, adrenal, and gonadal function. Barker et al. ${ }^{12}$ showed that excessive weight gain during childhood, in children with low BW, predicts future risk of developing coronary artery disease (CAD). In 1993, for the first time the association of low BW and adult hyperinsulinemia was presented in a retrospective study. ${ }^{13}$ Since then, insulin resistance in children and adults born with SGA condition have been reported, while insulin resistance in this group had no association with factors such as body mass index (BMI) and age. ${ }^{14-17}$ For the babies with the LGA condition, there is increased incidence of cesarean birth, shoulder dystocia, neonatal hypoglycemia, and longer stay in the hospital. ${ }^{18}$ Other side effects of the macrosomia include increased incidence of intra-uterine mortality, bone fracture, neonatal hyperbilirubinemia, brachial nerve paralysis, and obesity in childhood and adolescence. ${ }^{19}$

We investigated the associations between anthropometric characteristics and insulin markers in healthy mothers and the BW of their healthy term neonates. Also, possible associations between serum insulin markers of mothers and those of their neonates were explored.

\section{Material and Methods}

This observational study was carried out in a hospital in Tehran, Iran in 2014. The cohort consisted of 156 healthy pregnant women followed until their delivery time as well as their singleton newborns. Between 24-28 weeks of gestation the O'Sullivan glucose test was performed for all future mothers by ingesting $75 \mathrm{~g}$ of glucose and 38 mothers were excluded from the study for abnormal oral glucose tolerance test (OGTT). Other excluded data also consisted of 5 neonates with BW below $2,500 \mathrm{~g}$ as well as 3 neonates with BW over 4,000 g since for this study, only newborns with AGA status were included. After incomplete data and outliers were removed, 100 mothers and their babies comprised the study sample.

Pregnant women (18 to 40 years) the gestational age (GA) between 38 to 41 weeks determined by sonography and last menstrual period (LMP) and their neonates were studied. Mothers with unclear health history or the possible presence of the following problems were excluded: diabetes, gestational diabetes mellitus (GDM), previous history of GDM, hypertension, preeclampsia, and any other condition that affects serum glucose level, apparent malnourishment, smoking, and alcohol and drug abuse.

Data (the type of delivery, parity, mother's age, GA, weight before pregnancy and at delivery, weight-gain during pregnancy, and height) was collected by a trained midwife. Data for weightbefore pregnancy was collected from the most recent medical records before pregnancy and data for weight-at-delivery was collected right before delivery time. Weight-gain was calculated by subtracting weight-before pregnancy from weight-at-delivery. Data for anthropometric measurements of the neonates were recorded right after birth.

\section{Laboratory methods}

The fasting-blood-samples were obtained from mothers at delivery time and for neonates, the blood was collected through puncture of the umbilical cord artery in anticoagulant-free tubes and sent to the laboratory immediately. The blood samples were centrifuged at 3,000 rpm for 10-15 minutes and the obtained serum was frozen at $-70^{\circ} \mathrm{C}$ until assays for fasting glucose and insulin were performed. Serum glucose levels were assayed by an enzymatic colorimetric method with glucose oxidase using commercial kits (Pars Azmon Inc., Tehran, Iran) and a Selectra 2 auto-analyzer (Vital Scientific, Pankeren, the Netherlands). The intra- and inter-assay coefficients of variation $(\mathrm{CV})$ were $1.4 \%$ and $1.5 \%$, respectively.

Fasting serum insulin concentrations were 
Table I. Demographic and Biochemical Data for 100 Pregnant Women.

\begin{tabular}{lll}
\hline Variables & Mean \pm SD & Range \\
\hline Age (years) & $30 \pm 7$ & $23-43$ \\
BMI before pregnancy $\left(\mathrm{kg} / \mathrm{m}^{2}\right)$ & $22 \pm 3.8$ & $17.5-26.8$ \\
Weight before pregnancy $(\mathrm{kg})$ & $55 \pm 9.5$ & $45-73$ \\
Weight at delivery $(\mathrm{kg})$ & $68 \pm 8.3$ & $56-83$ \\
Weight gain during pregnancy $(\mathrm{kg})$ & $13.5 \pm 4.4$ & $10-20$ \\
Height $(\mathrm{cm})$ & $160 \pm 6.2$ & $151-171$ \\
Glucose $(\mathrm{mg} / \mathrm{dl})$ & $95.6 \pm 28.6$ & $64-140$ \\
Insulin $(\mathrm{mIU} / \mathrm{ml})$ & $13.9 \pm 3.6$ & $9.3-19.8$ \\
HOMA-IR & $3.3 \pm 1.4$ & $1-5.5$ \\
HOMA-S & $0.3 \pm 0.1$ & $0.3-0.5$ \\
QUICKI & $0.3 \pm 0.02$ & $0.2-0.4$ \\
GI ratio (mg/10-4 U) & $7.2 \pm 2.4$ & $3.2-10.8$ \\
\hline
\end{tabular}

BMI: body mass index; GI ratio: glucose/insulin ratio.; HOMA-IR: homeostatic model assessment for insulin resistance; HOMA-S: homeostatic model assessment for insulin sensitivity; QUICKI: quantitative insulin sensitivity check index

Table II. Demographic and Biochemical Data for 100 Full-Term Babies.

\begin{tabular}{lll}
\hline Variables & Mean \pm SD & Range \\
\hline Birth weight (g) & $3,224 \pm 346$ & $2,650-3,700$ \\
Gestational age (days) & $268 \pm 9$ & $256-280$ \\
Glucose (mg/dl) & $73.5 \pm 20.1$ & $43-118$ \\
Insulin (mIU/ml) & $6.7 \pm 4.5$ & $0.9-17.7$ \\
HOMA-IR & $1.2 \pm 0.9$ & $0.1-3.3$ \\
HOMA-S & $1.8 \pm 2.5$ & $0.3-10.8$ \\
QUICKI & $0.4 \pm 0.07$ & $0.3-0.6$ \\
GI ratio $\left(\mathrm{mg} / 10^{-4} \mathrm{U}\right)$ & $19.4 \pm 17.5$ & $4.3-55.7$
\end{tabular}

BMI: body mass index; GI ratio: glucose/insulin ratio.; HOMA-IR: homeostatic model assessment for insulin resistance; HOMA-S: homeostatic model assessment for insulin sensitivity; QUICKI: quantitative insulin sensitivity check index

measured by the electro-chemiluminescence immunoassay, the Roche/Hitachi Cobas e-411 analyzer and related kits $(\mathrm{GmbH}$, Mannheim, Germany). To monitor accuracy of assays, lyophilized quality control material (Lyphochek Immunoassay Plus Control, BioRad Laboratories) was used; intra- and interassay CVs were $1.3 \%$ and $1.4 \%$, respectively.

The insulin sensitivity and resistance were assessed by homeostatic model assessment (HOMA) models or HOMA-S for sensitivity and HOMA-IR for resistance.

Quantitative insulin sensitivity check index (QUICKI), and GI ratio in mothers and newborns were calculated by the following formulas:

HOMA-IR $=$ [Insulin $(\mathrm{mIU} / \mathrm{L}) *$ Glucose $(\mathrm{mmol} / \mathrm{L})]$ / 22.5; HOMA-S=1/HOMA-IR;

QUICKI $=1 /[\log$ Insulin $(\mathrm{mIU} / \mathrm{L})+\log$ Glucose $(\mathrm{mg} / \mathrm{dl})]$; and GI ratio= Glucose/ Insulin $\left(\mathrm{mg} / 10^{-4} \mathrm{U}\right)$.

The protocol of this study was approved by the Ethics Committee of the related medical university (36EC-RIES- 92/07/23) and was funded by the Research Institute for Endocrine Sciences (RIES). Mothers' informed consents were obtained.

\section{Statistical analyses}

The sample size for the study was determined by the formula, $\frac{\mathrm{n}=\left(\mathrm{z}^{\alpha} /\right)^{2} \sigma^{2}}{\mathrm{E}^{2}}$ where the confiden ce level was set at $95 \%$, the margin of error (E) was $8 \%$ (a larger $\mathrm{E}$ was chosen due to limited logistics), and standard deviation $(\sigma)$ was 0.5. Based on this formula, at least 150 


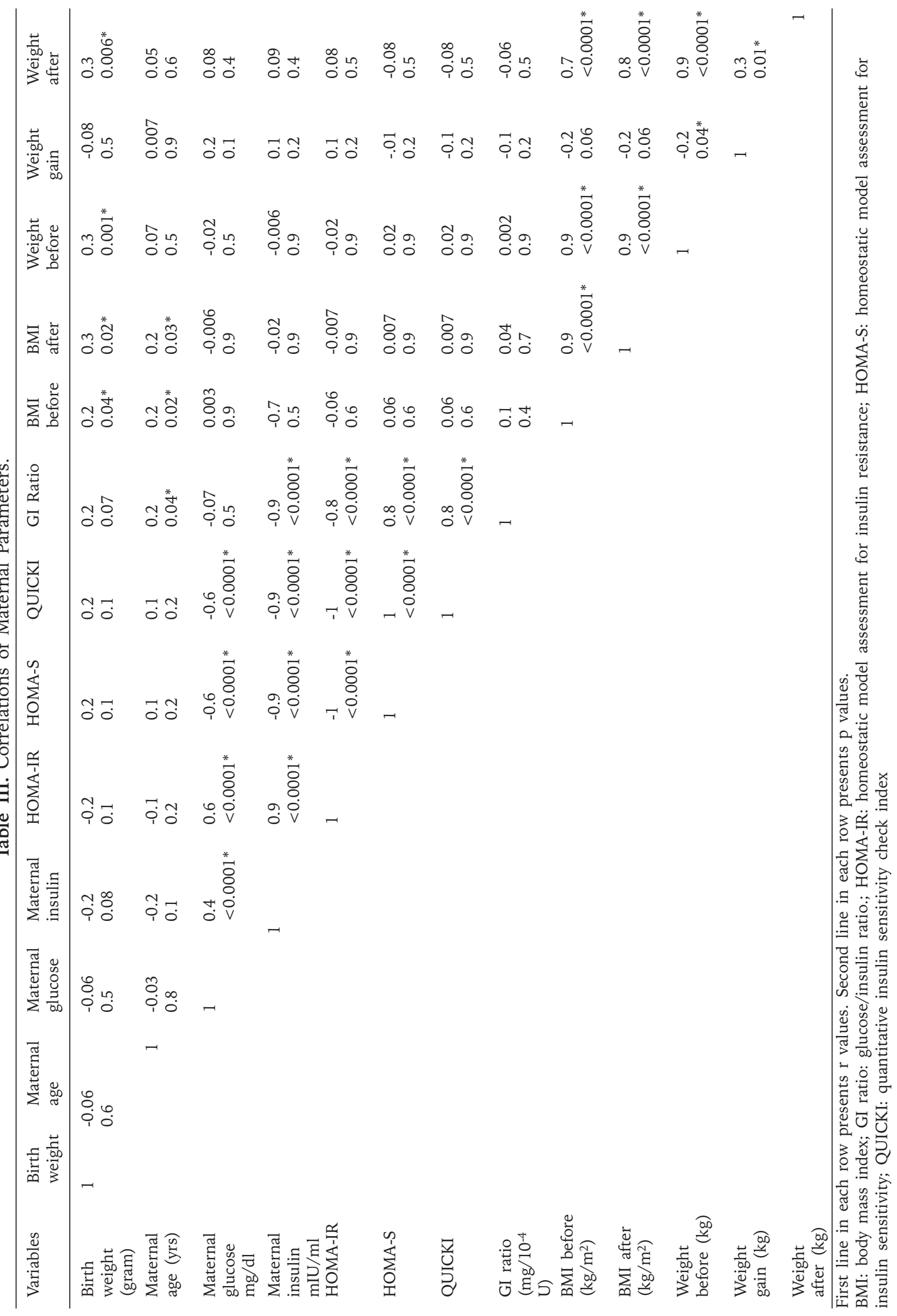


Table IV. Correlations Between Neonatal Variables.

\begin{tabular}{|c|c|c|c|c|c|c|c|c|}
\hline Variables & Glucose & Insulin & HOMA-IR & HOMA-S & QUICKI & GI ratio & $\begin{array}{l}\text { Birth } \\
\text { weight }\end{array}$ & $\begin{array}{l}\text { Gestational } \\
\text { age }\end{array}$ \\
\hline Glucose $(\mathrm{mg} / \mathrm{dl})$ & 1 & $\begin{array}{l}0.3 \\
0.006^{*}\end{array}$ & $\begin{array}{l}0.6 \\
<0.0001^{*}\end{array}$ & $\begin{array}{l}-0.6 \\
<0.0001^{*}\end{array}$ & $\begin{array}{l}-0.5 \\
<0.0001^{*}\end{array}$ & $\begin{array}{l}0.08 \\
0.4\end{array}$ & $\begin{array}{l}0.02 \\
0.9\end{array}$ & $\begin{array}{l}0.07 \\
0.5\end{array}$ \\
\hline Insulin $(\mathrm{mIU} / \mathrm{ml})$ & & 1 & $\begin{array}{l}0.9 \\
<0.0001^{*}\end{array}$ & $\begin{array}{l}-0.9 \\
<0.0001^{*}\end{array}$ & $\begin{array}{l}-0.9 \\
<0.0001^{*}\end{array}$ & $\begin{array}{l}-0.9 \\
<0.0001^{*}\end{array}$ & $\begin{array}{l}-0.01 \\
0.9\end{array}$ & $\begin{array}{l}-0.2 \\
0.03^{*}\end{array}$ \\
\hline HOMA-IR & & & 1 & $\begin{array}{l}-1 \\
<0.0001^{*}\end{array}$ & $\begin{array}{l}-1 \\
<0.0001^{*}\end{array}$ & $\begin{array}{l}-0.7 \\
<0.0001^{*}\end{array}$ & $\begin{array}{l}0.04 \\
0.7\end{array}$ & $\begin{array}{l}-0.2 \\
0.09\end{array}$ \\
\hline HOMA-S & & & & 1 & $\begin{array}{l}1 \\
<0.0001^{*}\end{array}$ & $\begin{array}{l}0.7 \\
<0.0001^{*}\end{array}$ & $\begin{array}{l}-0.04 \\
0.7\end{array}$ & $\begin{array}{l}0.2 \\
0.09\end{array}$ \\
\hline QUICKI & & & & & 1 & $\begin{array}{l}0.7 \\
0.001^{*}\end{array}$ & $\begin{array}{l}-0.03 \\
0.7\end{array}$ & $\begin{array}{l}0.05 \\
0.6\end{array}$ \\
\hline $\begin{array}{l}\text { GI ratio } \\
\left(\mathrm{mg} / 10^{-4} \mathrm{U}\right)\end{array}$ & & & & & & 1 & $\begin{array}{l}0.09 \\
0.4\end{array}$ & $\begin{array}{l}0.2 \\
0.01 *\end{array}$ \\
\hline Birth weight (g) & & & & & & & 1 & $\begin{array}{l}0.2 \\
0.07\end{array}$ \\
\hline $\begin{array}{l}\text { Gestational age } \\
\text { (days) }\end{array}$ & & & & & & & & 1 \\
\hline
\end{tabular}

First line in each row presents $r$ values. Second line in each row presents $\mathrm{p}$ values.

GI ratio: glucose/insulin ratio.; HOMA-IR: homeostatic model assessment for insulin resistance; HOMA-S: homeostatic model assessment for insulin sensitivity; QUICKI: quantitative insulin sensitivity check index

participant mothers were needed.

The Kolmogorov-Smirnov test was used to test the normal data distribution. As some values displayed non-normal distribution, the nonparametric tests were used for those. Pearson and Spearman correlations between variables were used for variables with normal and abnormal distributions, respectively. Percentage distribution between groups was compared by Chi-square or Fisher's exact tests. The quartiles of insulin were calculated for mothers and their neonates and the anthropometric and biochemical variables were compared within group and then between each group with others. One-way ANOVA has been used followed by Tukey post-hoc tests for values with normal distributions and the Mann-Whitney $U$ test was employed to compare non-parametric variables.

Data for maternal BMI were divided into four groups: thin, normal, overweight, and obese. All statistical tests were performed by using the SPSS software package version 20 and $\mathrm{p}<0.05$ was considered significant.

\section{Results}

Among 100 mothers (mean age $30 \pm 7$ years, $53(53 \%)$ were in their first-time pregnancy (nullipara) and $36(36 \%), 7(7 \%), 2(2 \%)$, and $2(2 \%)$ had second to fifth parity, respectively.
Among 100 neonates: 42 (42\%) were female and $58(58 \%)$ were male, $72(72 \%)$ had vaginal delivery, and $28(28 \%)$ had cesarean section (Tables I and II).

There was a positive correlation between maternal BMI before-pregnancy and neonate's weight $(r=0.2, p=0.04)$. Additionally, weight-before pregnancy $(\mathrm{r}=0.3, \mathrm{p}=0.001)$ and at-delivery $(\mathrm{r}=0.3, \mathrm{p}==0.006)$ showed positive correlation with BW. The maternal BMI at-delivery had also correlation with BW $(r=0.3, p=0.02)$ and maternal age $(r=$ $0.2, p=0.03)$. Maternal weight-gain during pregnancy negatively correlated with weightbefore pregnancy $(r=-0.2, p=0.04)$, and positively correlated with weight-at-delivery $(r=0.3, p=0.01)$. The final weight of mothers at-delivery showed a strong positive correlation with BMI before and after pregnancy $(r=0.7$ and $0.8, \mathrm{p}<0.0001$ ) (Table III).

With the neonates within the normal range for BW, no statistically significant correlation was found between BW and maternal or neonatal glucose, insulin, and markers of insulin sensitivity or resistance (Tables III and IV).

Among mothers, fasting serum glucose and insulin levels positively correlated with each other $(\mathrm{r}=0.4, \mathrm{p}<0.0001)$ and with HOMA-IR ( $\mathrm{r}=0.6, \mathrm{p}<0.0001)$, and negatively correlated with HOMA-S and QUICKI $(r=-0.6$, 
Table V. Comparison of Study Variables Based on Maternal Serum Insulin Quartiles.

\begin{tabular}{|c|c|c|c|c|c|c|}
\hline Variables & Q1 & Q2 & Q3 & Q4 & $\mathrm{P}$ value & $\begin{array}{l}\text { Post-hoc } p \\
\text { value }\end{array}$ \\
\hline $\begin{array}{l}\text { Maternal age } \\
\text { (years) }\end{array}$ & $\begin{array}{l}29 \pm 3.3 \\
(23-35)\end{array}$ & $\begin{array}{l}29 \pm 4.8 \\
(22-38)\end{array}$ & $\begin{array}{l}28 \pm 3.2 \\
(23-33)\end{array}$ & $\begin{array}{l}27 \pm 4 \\
(20-34)\end{array}$ & 0.2 & \\
\hline $\begin{array}{l}\text { Weight before } \\
\text { pregnancy }(\mathrm{kg})\end{array}$ & $\begin{array}{l}62 \pm 9.2 \\
(40-80)\end{array}$ & $\begin{array}{l}62 \pm 8.2 \\
(45-79)\end{array}$ & $\begin{array}{l}64 \pm 9.7 \\
(43-83)\end{array}$ & $\begin{array}{l}63 \pm 13.1 \\
(43-97)\end{array}$ & 0.9 & \\
\hline Height $(\mathrm{cm})$ & $\begin{array}{l}161 \pm 6.1 \\
(150-177)\end{array}$ & $\begin{array}{l}162 \pm 6.2 \\
(151-175)\end{array}$ & $\begin{array}{l}164 \pm 6.4 \\
(155-182)\end{array}$ & $\begin{array}{l}163 \pm 4.4 \\
(150-172)\end{array}$ & 0.4 & \\
\hline $\begin{array}{l}\text { Weight at } \\
\text { delivery }(\mathrm{kg})\end{array}$ & $\begin{array}{l}74 \pm 8.9 \\
(53-88)\end{array}$ & $\begin{array}{l}75 \pm 8.9 \\
(60-97.5)\end{array}$ & $\begin{array}{l}79 \pm 11.2 \\
(52-99)\end{array}$ & $\begin{array}{l}76 \pm 12.1 \\
(57-104)\end{array}$ & 0.4 & \\
\hline $\begin{array}{l}\text { BMI before } \\
\text { pregnancy } \\
\left(\mathrm{kg} / \mathrm{m}^{2}\right)\end{array}$ & $\begin{array}{l}24.5 \pm 4 \\
(16-33.3)\end{array}$ & $\begin{array}{l}23.6 \pm 3.4 \\
(17-30.1)\end{array}$ & $\begin{array}{l}23.8 \pm 3.8 \\
(15.8-34.5)\end{array}$ & $\begin{array}{l}23.5 \pm 4.9 \\
(14.2-34.4)\end{array}$ & 0.8 & \\
\hline $\begin{array}{l}\text { BMI at delivery } \\
\left(\mathrm{kg} / \mathrm{m}^{2}\right)\end{array}$ & $\begin{array}{l}24 \pm 3.9 \\
(15.6-33.3)\end{array}$ & $\begin{array}{l}23.5 \pm 3.4 \\
(16.9-30.1)\end{array}$ & $\begin{array}{l}23.8 \pm 3.8 \\
(15.8-34.6)\end{array}$ & $\begin{array}{l}23.7 \pm 4.4 \\
(17.9-34.4)\end{array}$ & 1.0 & \\
\hline $\begin{array}{l}\text { Weight } \\
\text { gain during } \\
\text { pregnancy }(\mathrm{kg})\end{array}$ & $\begin{array}{l}12.8 \pm 5.4 \\
(6.5-26)\end{array}$ & $\begin{array}{l}12.8 \pm 5.1 \\
(3-22)\end{array}$ & $\begin{array}{l}15.1 \pm 7.6 \\
(3-40)\end{array}$ & $\begin{array}{l}12.5 \pm 3.6 \\
(6-20)\end{array}$ & 0.3 & \\
\hline $\begin{array}{l}\text { Maternal glucose } \\
(\mathrm{mg} / \mathrm{dl})\end{array}$ & $\begin{array}{l}79 \pm 15.4 \\
(57-121)\end{array}$ & $\begin{array}{l}82.2 \pm 16.9 \\
(60-127)\end{array}$ & $\begin{array}{l}92.5 \pm 26 \\
(62-173)\end{array}$ & $\begin{array}{l}101.3 \pm 35.9 \\
(58-232)\end{array}$ & 0.004 & $\begin{array}{l}\text { Q1 vs. } \\
\text { Q4 }=0.005 \\
\text { Q2 vs. } \mathrm{Q} 4=0.03\end{array}$ \\
\hline $\begin{array}{l}\text { Maternal insulin } \\
(\mathrm{mIU} / \mathrm{L})\end{array}$ & $\begin{array}{l}5.6 \pm 2.1 \\
(0.7-8.4)\end{array}$ & $\begin{array}{l}10.9 \pm 1.4 \\
(8.4-12.8)\end{array}$ & $\begin{array}{l}18.2 \pm 3.3 \\
(12.8-23.39)\end{array}$ & $\begin{array}{l}40.9 \pm 18.8 \\
(23.3-103.2)\end{array}$ & $<0.0001$ & $\begin{array}{l}\text { Q1 vs. Q3 } \\
<0.0001 \\
\text { Q1 vs. Q4 } \\
<0.0001 \\
\text { Q2 vs. Q4 } \\
<0.0001 \\
\text { Q3 vs. Q4 } \\
<0.0001\end{array}$ \\
\hline $\begin{array}{l}\text { Maternal } \\
\text { HOMA-IR }\end{array}$ & $\begin{array}{l}1.1 \pm 0.4 \\
(0.1-1.8)\end{array}$ & $\begin{array}{l}2.2 \pm 0.5 \\
(1.4-2.9)\end{array}$ & $\begin{array}{l}4.2 \pm 1.3 \\
(2.4-7.5)\end{array}$ & $\begin{array}{l}11.2 \pm 8.7 \\
(3.5-45.5)\end{array}$ & $<0.0001$ & $\begin{array}{l}\text { Q1 vs. Q4 } \\
<0.0001 \\
\text { Q2 vs. } \\
\text { Q4<0.0001 } \\
\text { Q3 vs. } \\
\text { Q4<0.0001 }\end{array}$ \\
\hline $\begin{array}{l}\text { Maternal } \\
\text { HOMA-S }\end{array}$ & $\begin{array}{l}1.4 \pm 0.8 \\
(0.6-2.2)\end{array}$ & $\begin{array}{l}0.5 \pm 0.1 \\
(0.3-0.7)\end{array}$ & $\begin{array}{l}0.3 \pm 0.07 \\
(0.1-0.4)\end{array}$ & $\begin{array}{l}0.1 \pm 0.06 \\
(0.02-0.3)\end{array}$ & $<0.0001$ & $\begin{array}{l}\text { Q1 vs. Q2 } \\
=0.002 \\
\text { Q1 vs. Q3 } \\
<0.0001 \\
\text { Q1 vs. Q4 } \\
<0.0001\end{array}$ \\
\hline $\begin{array}{l}\text { Maternal } \\
\text { QUICKI }\end{array}$ & $\begin{array}{l}0.4 \pm 0.05 \\
(0.4-0.6)\end{array}$ & $\begin{array}{l}0.3 \pm 0.01 \\
(0.3-0.4)\end{array}$ & $\begin{array}{l}0.3 \pm 0.01 \\
(0.3-0.33)\end{array}$ & $\begin{array}{l}0.3 \pm 0.02 \\
(0.2-0.32)\end{array}$ & $<0.0001$ & $\begin{array}{l}\text { Q1 vs. Q2, Q3, } \\
\text { Q4 <0.0001 } \\
\text { Q2 vs. } \\
\text { Q3 }=0.005 \\
\text { Q2 vs. } \\
\text { Q4 }<0.0001 \\
\text { Q3 vs. } \\
\text { Q4 }=0.002\end{array}$ \\
\hline $\begin{array}{l}\text { Maternal GI } \\
\text { ratio (mg/10-4 } \\
\text { U) }\end{array}$ & $\begin{array}{l}19.6 \pm \\
18.7 \\
(7.3-90.9)\end{array}$ & $\begin{array}{l}7.7 \pm 2.2 \\
(4.9-14.3)\end{array}$ & $\begin{array}{l}5.3 \pm 2.1 \\
(2.8-13.5)\end{array}$ & $\begin{array}{l}2.7 \pm 0.9 \\
(1.4-5.5)\end{array}$ & $<0.0001$ & $\begin{array}{l}\text { Q1 vs. Q4 } \\
<0.0001 \\
\text { Q2 vs. } \\
\text { Q4<0.0001 } \\
\text { Q3 vs. } \\
\text { Q4<0.0001 }\end{array}$ \\
\hline
\end{tabular}




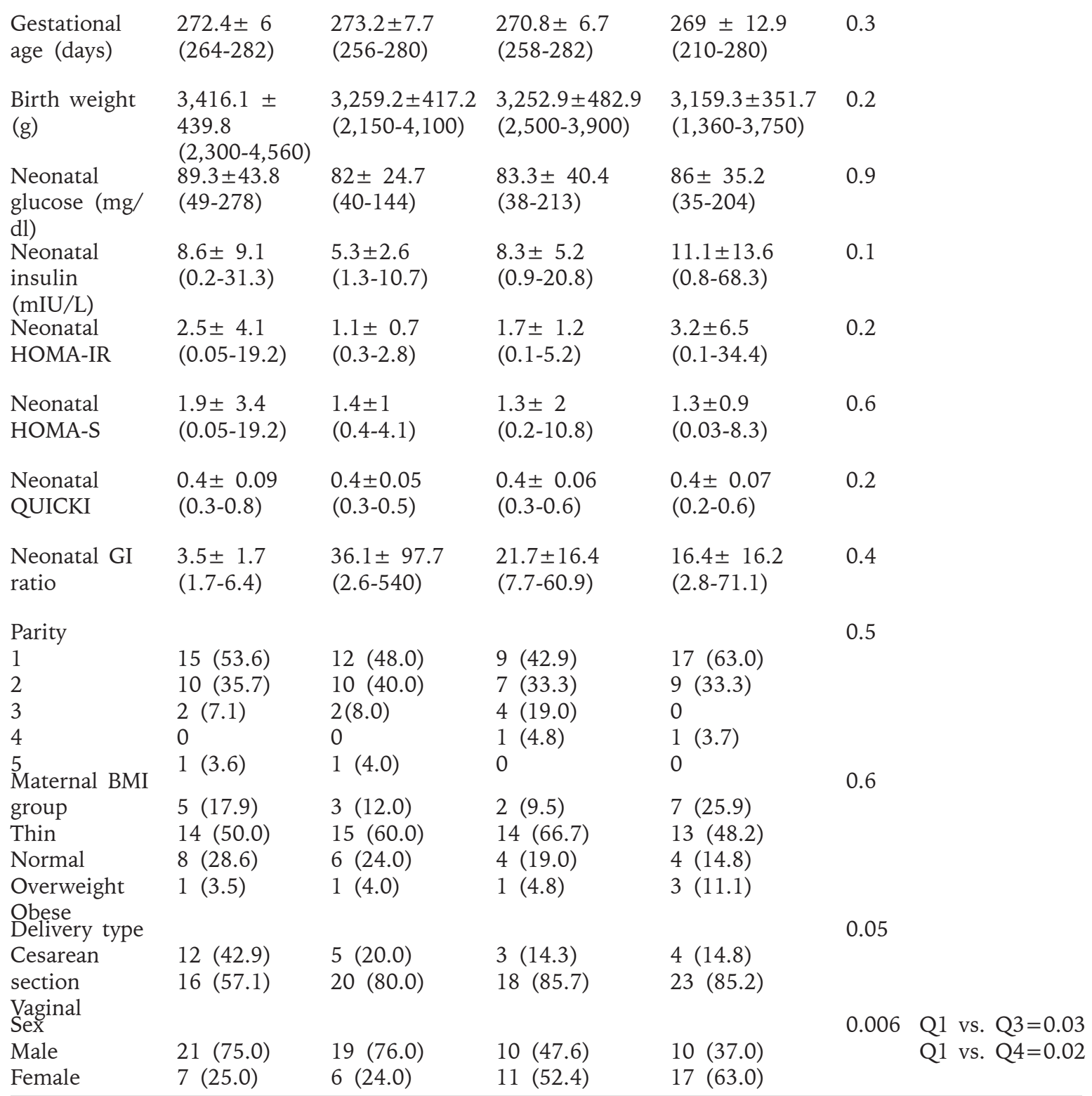

Data is presented as mean \pm SD (range) or $\mathrm{N}(\%)$, as appropriate.

HOMA IR: homeostatic model assessment for insulin resistance; HOMA-S: homeostatic model assessment for insulin sensitivity;

QUICKI: quantitative insulin sensitivity check index; GI ratio: glucose/insulin ratio.

$\mathrm{p}<0.0001)$. Serum insulin negatively correlated with insulin sensitivity markers and positively correlated with insulin resistance parameters $(\mathrm{p}<0.0001)$.

Similarly, in neonates serum glucose and insulin positively correlated with each other $(\mathrm{r}=0.3, \mathrm{p}=0.006)$ and with HOMA-IR ( $\mathrm{r}=$ $0.6, \mathrm{p}<0.0001)$ and negatively correlated with HOMA-S $(\mathrm{r}=-0.6, \mathrm{p}<0.0001)$ and QUICKI $(r=-0.5, p<0.0001)$. Neonates' serum insulin also negatively correlated with insulin sensitivity markers and positively with insulin resistance parameters $(p<0.0001)$. Serum insulin negatively $(\mathrm{r}=-0.2, \mathrm{p}=0.03)$ and $\mathrm{GI}$ ratio positively $(\mathrm{r}=0.2, \mathrm{p}=0.01)$ correlated with GA.

Quartiles of insulin for both mothers and neonates were analyzed (Table V and VI). The cutoff points for first and last quartiles were $5.6 \pm 2.1,40.9 \pm 18.8 \mathrm{mIU} / \mathrm{ml}$ in mothers, and 
Table VI. Comparison of Study Variables Based on Neonates' Insulin Quartiles.

\begin{tabular}{|c|c|c|c|c|c|c|}
\hline Variables & Q1 & Q2 & Q3 & Q4 & P Value* & $\begin{array}{l}\text { Post-hoc p } \\
\text { value* }\end{array}$ \\
\hline $\begin{array}{l}\text { Maternal age } \\
\text { (years) }\end{array}$ & $\begin{array}{l}29 \pm 4.2 \\
(20-38)\end{array}$ & $\begin{array}{l}27 \pm 3.1 \\
22-33\end{array}$ & $\begin{array}{l}29 \pm 4.3 \\
21-38\end{array}$ & $\begin{array}{l}28 \pm 4.9 \\
23-43\end{array}$ & 0.6 & \\
\hline $\begin{array}{l}\text { Weight before } \\
\text { pregnancy (kg) }\end{array}$ & $\begin{array}{l}64 \pm 11.5 \\
(40-97)\end{array}$ & $\begin{array}{l}62 \pm 10.8 \\
43-88.5\end{array}$ & $\begin{array}{l}62 \pm 9.8 \\
45-79\end{array}$ & $\begin{array}{l}59 \pm 8.7 \\
43-73\end{array}$ & 0.4 & \\
\hline Height $(\mathrm{cm})$ & $\begin{array}{l}163 \pm 4.7 \\
(155-170)\end{array}$ & $\begin{array}{l}163 \pm 6.1 \\
154-177\end{array}$ & $\begin{array}{l}160 \pm 5.5 \\
150-172\end{array}$ & $\begin{array}{l}162 \pm 7.1 \\
145-182\end{array}$ & 0.5 & \\
\hline $\begin{array}{l}\text { Weight at } \\
\text { delivery (kg) }\end{array}$ & $\begin{array}{l}75 \pm 11 \\
(53-104)\end{array}$ & $\begin{array}{l}75 \pm 11.5 \\
52-99\end{array}$ & $\begin{array}{l}76 \pm 10.4 \\
55-97.5\end{array}$ & $\begin{array}{l}72 \pm 8.2 \\
56-88\end{array}$ & 0.6 & \\
\hline $\begin{array}{l}\text { BMI before } \\
\text { pregnancy }(\mathrm{kg} / \\
\left.\mathrm{m}^{2}\right)\end{array}$ & $\begin{array}{l}24 \pm 4 \\
(16-33.3)\end{array}$ & $\begin{array}{l}24 \pm 3.4 \\
17-30.1\end{array}$ & $\begin{array}{l}24 \pm 3.8 \\
15.8-34.5\end{array}$ & $\begin{array}{l}24 \pm 4.9 \\
14.2-34.4\end{array}$ & 0.8 & \\
\hline $\begin{array}{l}\text { BMI at delivery } \\
\left(\mathrm{kg} / \mathrm{m}^{2}\right)\end{array}$ & $\begin{array}{l}24 \pm 3.9 \\
(16-34)\end{array}$ & $\begin{array}{l}23 \pm 4.1 \\
15.8-33.7\end{array}$ & $\begin{array}{l}24 \pm 3 \\
16.9-30.1\end{array}$ & $\begin{array}{l}23 \pm 3.2 \\
17.6-30\end{array}$ & 0.4 & \\
\hline $\begin{array}{l}\text { Weight } \\
\text { gain during } \\
\text { pregnancy }(\mathrm{kg})\end{array}$ & $\begin{array}{l}13 \pm 5 \\
(5-26)\end{array}$ & $\begin{array}{l}14 \pm 7.1 \\
3-40\end{array}$ & $\begin{array}{l}14 \pm 4.8 \\
3-22\end{array}$ & $\begin{array}{l}13 \pm 3.7 \\
7-21\end{array}$ & 0.7 & \\
\hline $\begin{array}{l}\text { Maternal } \\
\text { glucose }(\mathrm{mg} / \mathrm{dl})\end{array}$ & $\begin{array}{l}92 \pm 36.2 \\
(57-232)\end{array}$ & $\begin{array}{l}92.4 \pm 28.5 \\
58-173\end{array}$ & $\begin{array}{l}82.9 \pm 14.1 \\
58-112\end{array}$ & $\begin{array}{l}97.2 \pm 25.6 \\
59-140\end{array}$ & 0.3 & \\
\hline $\begin{array}{l}\text { Maternal } \\
\text { insulin (mIU/L) }\end{array}$ & $\begin{array}{l}17.2 \pm 18 \\
(2-79.3)\end{array}$ & $\begin{array}{l}23.8 \pm 24.2 \\
0.7-103.2\end{array}$ & $\begin{array}{l}15.9 \pm 9.2 \\
4.4-36.9\end{array}$ & $\begin{array}{l}20.5 \pm 14.8 \\
4.8-86.7\end{array}$ & 0.4 & \\
\hline $\begin{array}{l}\text { Maternal } \\
\text { HOMA-IR }\end{array}$ & $\begin{array}{l}5 \pm 9 \\
(0.4-45.5)\end{array}$ & $\begin{array}{l}6.1 \pm 8.2 \\
0.1-39.8\end{array}$ & $\begin{array}{l}3.3 \pm 2.1 \\
0.8-8.7\end{array}$ & $\begin{array}{l}5.4 \pm 5 \\
0.7-22.7\end{array}$ & 0.5 & \\
\hline $\begin{array}{l}\text { Maternal } \\
\text { HOMA-S }\end{array}$ & $\begin{array}{l}0.7 \pm 0.6 \\
(0.02-2.4)\end{array}$ & $\begin{array}{l}0.8 \pm 2 \\
0.03-10.2\end{array}$ & $\begin{array}{l}0.4 \pm 0.3 \\
0.1-1.3\end{array}$ & $\begin{array}{l}0.4 \pm 0.3 \\
0.04-1.4\end{array}$ & 0.5 & \\
\hline $\begin{array}{l}\text { Maternal } \\
\text { QUICKI }\end{array}$ & $\begin{array}{l}0.3 \pm 0.05 \\
(0.2-0.5)\end{array}$ & $\begin{array}{l}0.3 \pm 0.07 \\
0.2-0.6\end{array}$ & $\begin{array}{l}0.3 \pm 0.03 \\
0.3-0.4\end{array}$ & $\begin{array}{l}0.3 \pm 0.04 \\
0.3-0.4\end{array}$ & 0.4 & \\
\hline $\begin{array}{l}\text { Maternal GI } \\
\text { ratio (mg/10-4 } \\
\text { U) }\end{array}$ & $\begin{array}{l}10.5 \pm 9 \\
(1.8-42)\end{array}$ & $\begin{array}{l}10.6 \pm 17.7 \\
1.4-91\end{array}$ & $\begin{array}{l}7 \pm 3.7 \\
1.9-16.7\end{array}$ & $\begin{array}{l}6.9 \pm 4.3 \\
2-18.7\end{array}$ & 0.4 & \\
\hline $\begin{array}{l}\text { Gestational age } \\
\text { (days) }\end{array}$ & $\begin{array}{l}274.1 \pm 5.2 \\
(265-280)\end{array}$ & $\begin{array}{l}270.2 \pm 13.7 \\
210-182\end{array}$ & $\begin{array}{l}271 \pm 6.4 \\
259-280\end{array}$ & $\begin{array}{l}270.5 \pm 6.8 \\
250-282\end{array}$ & 0.4 & \\
\hline $\begin{array}{l}\text { Birth weight } \\
\text { (g) }\end{array}$ & $\begin{array}{l}3,297 \pm 257.4 \\
(3,000-3,750)\end{array}$ & $\begin{array}{l}3,302 \pm \\
345.4 \\
2,650-3,920\end{array}$ & $\begin{array}{l}3,294 \pm 358.4 \\
2,530-3,900\end{array}$ & $\begin{array}{l}3,256 \pm 413.3 \\
2,650-3,900\end{array}$ & 1.0 & \\
\hline $\begin{array}{l}\text { Neonatal } \\
\text { glucose (mg/dl) }\end{array}$ & $\begin{array}{l}83.3 \pm 22.6 \\
(49-138)\end{array}$ & $\begin{array}{l}76.2 \pm 35 \\
35-213\end{array}$ & $\begin{array}{l}82.6 \pm 25.1 \\
38-150\end{array}$ & $\begin{array}{l}115.5 \pm 54.8 \\
55-278\end{array}$ & $<0.0001$ & $\begin{array}{l}\text { Q1 vs. } \\
\text { Q4<0.0001 } \\
\text { Q2 vs. } \\
\text { Q4<0.0001 } \\
\text { Q3 vs. } \\
\text { Q4<0.0001 }\end{array}$ \\
\hline $\begin{array}{l}\text { Neonatal } \\
\text { insulin (mIU/L) }\end{array}$ & $\begin{array}{l}2.4 \pm 0.9 \\
(0.2-3.7)\end{array}$ & $\begin{array}{l}4.8 \pm 0.6 \\
3.9-5.6\end{array}$ & $\begin{array}{l}7.8 \pm 1.6 \\
5.8-12\end{array}$ & $\begin{array}{l}28.2 \pm 18.4 \\
12.4-93.9\end{array}$ & $<0.0001$ & $\begin{array}{l}\text { Q1 vs. } \\
\text { Q4<0.0001 } \\
\text { Q2 vs. } \\
\text { Q4<0.0001 } \\
\text { Q3 vs. } \\
\text { Q4<0.0001 }\end{array}$ \\
\hline
\end{tabular}




\begin{tabular}{|c|c|c|c|c|c|c|}
\hline $\begin{array}{l}\text { Neonatal } \\
\text { HOMA-IR }\end{array}$ & $\begin{array}{l}0.5 \pm 0.3 \\
(0.05-1.2)\end{array}$ & $\begin{array}{l}0.9 \pm 0.4 \\
0.4-2.6\end{array}$ & $\begin{array}{l}1.6 \pm 0.6 \\
0.7-1.2\end{array}$ & $\begin{array}{l}9.1 \pm 9.3 \\
2.1-37.6\end{array}$ & $<0.0001$ & $\begin{array}{l}\text { Q1 vs. } \\
\text { Q4<0.0001 } \\
\text { Q2 vs. } \\
\text { Q4 }<0.0001 \\
\text { Q3 vs. } \\
\text { Q4 }<0.0001\end{array}$ \\
\hline $\begin{array}{l}\text { Neonatal } \\
\text { HOMA-S }\end{array}$ & $\begin{array}{l}3.1 \pm 3.6 \\
(0.9-18.8)\end{array}$ & $\begin{array}{l}1.3 \pm 0.5 \\
0.4-2.6\end{array}$ & $\begin{array}{l}1.6 \pm 0.6 \\
0.7-1.2\end{array}$ & $\begin{array}{l}0.2 \pm 0.1 \\
0.03-0.5\end{array}$ & $<0.0001$ & $\begin{array}{l}\text { Q1 vs. } \\
\text { Q4<0.0001 } \\
\text { Q1 vs. } \\
\text { Q3 }<0.0001 \\
\text { Q1 vs. } \\
\text { Q4 }<0.0001\end{array}$ \\
\hline $\begin{array}{l}\text { Neonatal } \\
\text { QUICKI }\end{array}$ & $\begin{array}{l}0.5 \pm 0.07 \\
(0.4-0.8)\end{array}$ & $\begin{array}{l}0.4 \pm 0.03 \\
0.3-0.5\end{array}$ & $\begin{array}{l}0.4 \pm 0.02 \\
0.3-0.4\end{array}$ & $\begin{array}{l}0.3 \pm 0.03 \\
0.2-0.3\end{array}$ & $<0.0001$ & $\begin{array}{l}\text { Q1 vs. Q2, } \\
\text { Q1 vs. } \\
\text { Q3, Q1 vs. } \\
\text { Q4<0.0001; } \\
\text { Q2 vs. Q4, } \\
\text { Q3 vs. Q4 } \\
<0.0001 \\
\text { Q2 vs. } \\
\text { Q3 }=0.02\end{array}$ \\
\hline $\begin{array}{l}\text { Neonatal GI } \\
\text { ratio }\end{array}$ & $\begin{array}{l}56.2 \pm 101.8 \\
(15-540)\end{array}$ & $\begin{array}{l}16 \pm 7.7 \\
7.1-43.6\end{array}$ & $\begin{array}{l}10.8 \pm 3.4 \\
5.5-18.4\end{array}$ & $\begin{array}{l}4.8 \pm 2.3 \\
1.7-10.9\end{array}$ & 0.002 & $\begin{array}{l}\text { Q1 vs. Q2 } \\
=0.03 \\
\text { Q1 vs. } \\
\text { Q3 }=0.01 \\
\text { Q1 vs. } \\
\text { Q4 }=0.003\end{array}$ \\
\hline $\begin{array}{l}\text { Parity } \\
1 \\
2 \\
3 \\
4 \\
5\end{array}$ & $\begin{array}{ll}11 & (44.0) \\
12 & (48.0) \\
1 & (4.0) \\
1 & (4.0) \\
0 & \end{array}$ & $\begin{array}{l}17(68.0) \\
7(28.0) \\
0 \\
1(4.0) \\
0\end{array}$ & $\begin{array}{l}10(40.0) \\
10(40.0) \\
3(12.0) \\
0 \\
2(8.0)\end{array}$ & $\begin{array}{ll}14 & (56.0) \\
7 & (28.0) \\
3 & (12.0) \\
0 & \\
1 & (4.0)\end{array}$ & 0.3 & \\
\hline $\begin{array}{l}\text { Maternal BMI } \\
\text { group } \\
\text { Thin } \\
\text { Normal } \\
\text { Overweight } \\
\text { Obese }\end{array}$ & $\begin{array}{l}3(12) \\
14(56) \\
6(24) \\
2(8)\end{array}$ & $\begin{array}{l}6(24.0) \\
12(48.0) \\
5(20.0) \\
2(8.0)\end{array}$ & $\begin{array}{l}3(12) \\
15(60) \\
6(24) \\
1(4)\end{array}$ & $\begin{array}{l}7(28) \\
14(56) \\
4(16) \\
0\end{array}$ & 0.5 & \\
\hline $\begin{array}{l}\text { Delivery type } \\
\text { Cesarean } \\
\text { section } \\
\text { Vaginal }\end{array}$ & $\begin{array}{l}5(20.0) \\
20(80.0)\end{array}$ & $\begin{array}{l}5(20.0) \\
20(80.0)\end{array}$ & $\begin{array}{l}7(28.0) \\
18(72.0)\end{array}$ & $\begin{array}{ll}11 & (44.0) \\
14 & (56.0)\end{array}$ & 0.2 & \\
\hline $\begin{array}{l}\text { Sex } \\
\text { Male } \\
\text { Female }\end{array}$ & $\begin{array}{l}17(68.0) \\
8(32.0)\end{array}$ & $\begin{array}{ll}13 & (52.0) \\
12 & (48.0) \\
\end{array}$ & $\begin{array}{l}17(68.0) \\
8(32.0)\end{array}$ & $\begin{array}{l}11(44.0) \\
14(56.0) \\
\end{array}$ & 0.2 & \\
\hline
\end{tabular}

Data is presented as mean $\pm \mathrm{SD}$ (range) or $\mathrm{N}(\%)$, as appropriate.

HOMA IR: homeostatic model assessment for insulin resistance; HOMA-S: homeostatic model assessment for insulin sensitivity;

QUICKI: quantitative insulin sensitivity check index; GI ratio: glucose/insulin ratio.

$2.4 \pm 0.9,28.2 \pm 18.4 \mathrm{mIU} / \mathrm{ml}$ in neonates. Neonatal glucose, HOMA-IR, HOMA-S, QUICKI, and GI ratio in different neonatal insulin quartiles showed statistically significant differences (Table VI)
Maternal insulin quartiles showed similar results. Maternal weight and BMI-beforepregnancy, at-delivery, and weight-gain during pregnancy had no statistically significant differences in different maternal insulin quartiles (Table V). 
Additionally, first maternal insulin quartile had dominantly male and last quartile had dominantly female babies $(\mathrm{p}=0.006)$. There were no statistically significant differences for GA or BW in maternal insulin quartiles $(\mathrm{p}=0.2) \quad($ Table $\mathrm{V})$.

In different neonatal insulin quartiles, no statistically significant differences for maternal and biochemical parameters, BW, GA, and sex were found (Table VI), but quartile with the highest neonatal insulin had higher glucose, HOMA-IR but lower HOMA-S, QUICKI $(p<0.0001)$, and GI ratio $(p=0.002)$ compared to the lowest insulin quartile.

\section{Discussion}

Findings of this study indicate that neonate's BW was positively affected by maternal weight and BMI before pregnancy and at the time of delivery. The study evaluated if there was any correlation between maternal serum insulin level and the baby's BW. Within our BW range $(2,650-3,700$ grams) our $p$ value did not reach to statistical significance. Future studies on larger sample populations may further explore these correlations to increase the generalizability of the outcomes. ${ }^{20}$

Other researchers have shown that mothers' central obesity correlates with babies' hyperinsulinemia. ${ }^{21}$ Although maternal central obesity was not measured in this study, and instead, maternal weight and BMI were used, when we compared neonatal insulin quartiles, no statistically significant difference for maternal weight or BMI between quartiles was identified. The incidence of insulin resistance has been reported higher in LGA group compared to AGA babies. ${ }^{22,23}$ Yet, we found no statistically significant differences for BW among insulin quartiles since our sample included only AGA neonates.

Although the incidence of metabolic and endocrine disorders in newborns with abnormal BW is higher, sufficient knowledge is not available to suggest that endocrine interventions can be a solution to reduction in the SGA incidences. ${ }^{22}$ Controlling maternal weight may provide a better approach in controlling the infants' weight.

Lower GI ratios in babies associate with lower HOMA-S and QUICKI and higher HOMA-IR. ${ }^{23}$
Our results were in line with the above findings. We showed that HOMA-IR was negatively and HOMA-S and QUICKI were positively associated with GI ratio.

Mothers who were born with SGA condition have higher incidence of developing GDM. ${ }^{24,25}$ Since we excluded mothers with GDM from the study, we cannot explain the above correlations and within our range of serum sugar level for mothers (64-140 mg/dl), we could not show any association between mothers' serum glucose level and the infant's BW.

Other researchers have shown that male gender was associated with increased risk of GDM in mothers. ${ }^{26}$ However, our data showed that maternal serum glucose and insulin positively correlated with each other, and, the maternal quartile with the lowest insulin associated with predominance of male gender while maternal quartile with the highest insulin was mostly associated with female gender.

Study limitations included: the reference technique for assessing IR is hypo-insulinemic euglycemic clamp that is an expensive, complicated, and cumbersome method. ${ }^{27}$ So, we used HOMA-IR index. Also, the number of study population was modest because of lost to follow-up and exclusion of outlier data.

In conclusion, maternal anthropometric measures correlate with neonates' birth weight. Our results suggest that health promotion to normalize maternal weight parameters may better control the incidence of abnormal birth weights among newborns.

\section{Acknowledgement}

We would like to thank mothers who agreed to participate with their newborns in this study.

\section{REFERENCES}

1. Darendeliler F, Çoban A, Baş F, et al. Catch-up growth in appropriate- or small-for-gestational age preterm infants. Turk J Pediatr 2008; 50: 207-213.

2. Lee PA, Chernausek SD, Hokken-Koelega ACS, Czernichow P. International Small for Gestational Age Advisory Board Consensus Development Conference Statement: Management of short children born small for gestational age, April 24-October 1, 2001. Pediatrics 2003; 111: 1253-1261.

3. Rapoport R, Tuvemo T. Growth and growth hormone in children born small for gestational age. Acta Paediatr 2005; 94: 1348-1355. 
4. Hediger ML, Orerpeck MD, Maurer KR, KuczMarski RJ, McGlynr A, Davis WW. Growth of infants and young children born small or large for gestational age: Findings from the third National Health and Nutrition Examination survey. Arch Pediatric Adolesc Med 1998; 152: $1225-1231$

5. Li C Johonson MS, Goran MI. Effects of low birth weight on insulin- resistance syndrome in Caucasian and African-American children. Diabetes Care 2001; 24: 2035-2042.

6. Wen SW, Kramer MS, Usher RH. Comparison of birth weight distributions between Chinese and Caucasian infants. Am J Epidemiol 1995; 41: 1177-1187.

7. Faienza MF, Marzano F, Ventura AM, et al. Regulation of IGFBP3 gene expression in short children born small for gestational age. Growth Horm IGF Res 2011; 21: 349-355.

8. Gluckman PD, Hanson MA. The consequences of being born small - an adaptive perspective. Horm Res 2005; 65: 5-14.

9. Jovanovic L. A tincture of time does not turn the tide: Type 2 diabetes trends in offspring of type 2 diabetic mothers. Diabetes Care 2000; 23: 1219-1220.

10. Barker DJ, Osmond C, Forsen TJ, Kajantie E, Eriksson JG. Trajectories of growth among children who have coronary events as adults. N Engl J Med 2005; 353: 1802-1809.

11. Stettler N, Stalling VA, Troxel AB, et al. Weight gain in the first week of life and overweight in adulthood: A cohort study of European American subjects fed infant formula. Circulation 2005; 111: 1897-1903.

12. Barker DJ, Osmond C, Forsen TJ, Kajantie E, Eriksson JG. Trajectories of growth among children who have coronary events as adults. N Eng J Med 2005; 353: 802-809.

13. Chen $\mathrm{M}, \mathrm{Wu} \mathrm{L}, \mathrm{Wu} F$, et al. Impaired glucose metabolism in response to high fat diet in female mice conceived by in vitro fertilization (IVF) or ovarian stimulation alone. PLoS One 2014; 9: e113155.

14. Juqvet D, Gabariau A, Czernichow P, Levy-Marchal C. Insulin resistance early in adulthood in subjects born with intra uterine growth retardation. J Clin Endocrinol Metab 2000; 85: 1401-1406.

15. Flanagan DE, Moore VM, Godsland IF, Cockington RA, Robinson JS, Phillips DI. Fetal growth and the physiological control of glucose tolerance in adults: A minimal model analysis. Am J Physiol Endocrinol Metab 2000; 278: 700-706.
16. Veening MA, Van Weissinbruch MM, Delemmarre-Van De Waal HA. Glucose tolerance, insulin sensitivity, and insulin secretion in children born small for gestational age. J Clin Endocrinol Metab 2002; 87: 4557-4551.

17. Hofman PL, Cutfield WS, Robinson EM, et al. Insulin Resistance in short children with intrauterine growth retardation. J Clin Endocrinol Metab 1997; 82: 402406.

18. Weissmann-Brenner A, Simchen MJ, Zilberberg E, et al. Maternal and neonatal outcomes of macrosomic pregnancies. Med Sci Monit 2012; 18: 77-81.

19. Costa BM, Paulinelli RR, Borhosa MA. Association between maternal and fetal weight gain: Cohort study. Sao Paulo Med J 2012; 130: 242-7.

20. Nourbakhsh S, Ashrafzadeh S, Hafizi A, Naseh A. Associations between maternal anthropometric characteristics and infant birth weight in Iranian population. Sage Open Med 2016; 4: 2050312116646691.

21. Yejnik CS, Glubree H, Rege SS, et al. Adiposity and hyperinsulinemia in Indians are present at birth. J Clin Endocrinol Metab 2002; 87: 5575-5580.

22. Clayton PE, Cianfarani S, Czernichow P, Johannsson G, Rapaport R, Rogol A. Management of the child born small for gestational age through adulthood: A consensus statement of the International Societies of Pediatric Endocrinology and the Growth Hormone Research Society. J Clin Endocrinol Metab 2007; 92: 804-810.

23. Gesteiro E, Bastida S, Sánchez-Muniz FJ. Insulin resistance markers in term normoweight neonates. The Merida Cohort. Eur J Pediatrics 2009; 188: 281-288.

24. Innes KE, Byer TE, Marshall YA, Boron A, Orleans M, Hamman RF. Association of a woman's own birth weight with subsequent risk for gestational diabetes. JAMA 2002; 287: 2534-41.

25. Wallace TM, Levy JC, et al. Use and Abuse of HOMA modeling. Diabetes Care 2004; 27: 1487-95.

26. Retnakaran R. Fetal sex and maternal risk of gestational diabetes mellitus: The impact of having a boy. Diabetes Care 2015; 35: 844-851.

27. Muniyappa R. Current approaches for assessing insulin sensitivity and resistance in vivo: Advantages, limitations, and appropriate usage. Am J Physiol Endocrinol Metab 2008; 294: 15-26. 